\title{
Modelo validado para predecir el riesgo cardiovascular en adultos jóvenes según estilo de vida
}

\author{
Validated model to predict cardiovascular risk in young adults according to lifestyle
}

Goodling HC y col. JAMA Intern Med. 2017;177(9):1354-60

\section{Objetivos}

Evaluar el desempeño de una escala basada en el estilo de vida para predecir la aparición de eventos cardiovasculares (CV) antes de los 55 años, en adultos jóvenes.

\section{Diseño, lugar y participantes}

Estudio de cohorte prospectiva, iniciado en 1985, que incluyó a 4893 adultos de 18 a 30 años del estudio CARDIA ${ }^{1}$ (Coronary Artery Disease Risk Development in Young Adults) provenientes de cuatro centros en Estados Unidos. Los participantes (54,9\% mujeres, 50,7\% afroamericanos) no tenían antecedentes de enfermedad CV, cáncer u otras enfermedades crónicas y se les determinaron la tensión arterial, la glucemia en ayunas y el perfil lipídico.

\section{Factores pronósticos}

Se aplicó a la cohorte una escala de riesgo CV derivada y validada previamente en otra población (Healthy Heart Score -o $\mathrm{HHS})^{2}$. La escala incluye las siguientes variables: sexo, edad, índice de masa corporal, tabaquismo, consumo de alcohol, actividad física y dieta (consumo de frutas y verduras, fibra proveniente de cereales integrales, frutos secos, carnes rojas y procesadas y bebidas azucaradas). Se calculó el riesgo CV de los pacientes a 20 años, en base a sus respuestas basales.

\section{Medición de resultados principales}

Capacidad del HHS para predecir el riesgo a 25 años de eventos CV mayores: muerte por enfermedad coronaria, infarto de miocardio no fatal y accidente cerebrovascular isquémico fatal o no fatal en la muestra total, en subgrupos específicos de raza y sexo, con y sin factores de riesgo.

Se evaluó la discriminación del HHS mediante el estadístico C de Harrell y la calibración, mediante gráficos ${ }^{3}$.

\section{Resultados principales}

La mediana de seguimiento fue de 27,1 años. La incidencia de eventos CV mayores fue 0,9 casos/1000 personas-año en mujeres y 1,75 casos/1000 personas año en varones. Los participantes que sufrieron un evento CV tenían en promedio niveles menos saludables de HHS y mayores niveles de tensión arterial sistólica y diastólica, glucemia en ayunas y colesterol total que los participantes que no sufrieron eventos.

La discriminación del HHS fue moderada: estadístico C 0,71 (IC $95 \% 0,66$ a 0,76 ). Fue mayor en participantes de etnia blanca, en varones y en pacientes sin factores de riesgo basales. Se observó un aumento leve de la discriminación al adaptar los coeficientes beta originales a la población en estudio: estadístico C 0,73 (IC $95 \% 0,68$ a 0,77 ). La calibración fue adecuada para la cohorte global, así como para todos los subgrupos.

\section{Conclusiones}

EI HHS es moderadamente útil para predecir la aparición de eventos CV prematuros en adultos jóvenes, especialmente en aquellos sin factores de riesgo adicionales.

Fuentes de financiamiento: NHLBI, NIH. Conflictos de interés: No referidos.

\section{Comentario}

La principal fortaleza de la escala HHS consiste en su habilidad para estimar el riesgo CV a largo plazo en adultos jóvenes sin factores de riesgo, en base a características de su estilo de vida. Tiene una moderada precisión, similar al de otras herramientas que se utilizan habitualmente en la práctica clínica, como la escala de riesgo de Framingham ${ }^{3}$ (estadístico de $\mathrm{C}$ de 0,75 a 0,80 , dependiendo de la población). Es una herramienta fácil de utilizar y evalúa en forma exhaustiva aspectos de la dieta y de la actividad física que pueden ser pasados por alto en un interrogatorio no estructurado. Si bien existen recomendaciones universales acerca de la alimentación y la actividad física, que pueden ser puestas en práctica en toda la población, una posible aplicación del HHS es identificar a los pacientes con mayor riesgo cardiovascular en base a sus hábitos, los cuales se podrían beneficiar con la participación en programas intensivos de modificación del estilo de vida. Existe evidencia de un beneficio pequeño de estos programas en personas adultas de cualquier edad sin factores de riesgo CV (recomendación de tipo opcional para la USPSTF) ${ }^{4}$ y un beneficio mayor en adultos con sobrepeso u obesidad que además tengan otro factor de riesgo como hipertensión, diabetes o dislipemia (recomendación débil a favor ${ }^{5}$ ). Sin embargo, no existe actualmente evidencia de la efectividad del uso del HHS con este fin.

Este es un nuevo modelo de predicción clínica, que fue validado hasta el momento en sólo dos estudios. Se necesitan sumar más pruebas de su validez y estimar el impacto clínico de su implementación a nivel poblacional antes de recomendar su uso sistemático ${ }^{6}$.

\section{Conclusiones del comentador}

El HHS es una herramienta interesante para usar en la consejería y prevención primaria de eventos cardiovasculares en adultos jóvenes, que podría ser útil para identificar a los pacientes con mayor riesgo cardiovascular en base a sus hábitos. Sin embargo, la evidencia es insuficiente para avalar su utilización a nivel poblacional.

Sebastián Sguiglia [ Servicio de Medicina Familiar y Comunitaria, Hospital Italiano de Buenos Aires, sebastian.sguiglia@ hospitalitaliano.org.ar ]

Sguiglia S. Modelo validado para predecir el riesgo cardiovascular en adultos jóvenes según estilo de vida. Evid Actual Pract Ambul 2018;21(4):111. Comentado de: Gooding HC y col. Application of a Lifestyle-Based Tool to Estimate Premature Cardiovascular Disease Events in Young Adults: The Coronary Artery Risk Development in Young Adults (CARDIA) Study. JAMA Intern Med. 2017; 177(9):1354-1360. PMID: 28715555

\section{Referencias}

1. Friedman GD, y col. CARDIA: study design, recruitment, and some characteristics of the examined subjects. J Clin Epidemiol. $1988 ; 41(11): 1105-1116$. 2. Chiuve SE, y col. Lifestyle-based prediction model for the prevention of CVD: the Healthy Heart Score. J AmHeart Assoc. 2014;3(6):e000954.

3. Alba AC, y col. Discrimination and Calibration of Clinical Prediction Models. Users' Guides to the Medical Literature. JAMA. 2017;318(14):1377-1384.

4. US Preventive Services Task Force. Behavioral Counseling to Promote a Healthful Diet and Physical Activity for Cardiovascular Disease Prevention in Adults Without Cardiovascular Risk Factors US Preventive Services Task Force Recommendation Statement. JAMA. 2017;318(2):167-174.

5. LeFevre ML, on behalf of the U.S. Preventive Services Task Force. Behavioral Counseling to Promote a Healthful Diet and Physical Activity for Cardiovascular Disease Prevention in Adults With Cardiovascular Risk Factors: U.S. Preventive Services Task Force Recommendation Statement. Ann Intern Med. 2014;161:587-593

6. Toll DB,y col. Validation, updating and impact of clinical prediction rules: a review. J Clin Epidemiol 2008;61:1085-94 